\title{
Structure-guided design of next-generation malaria vaccine
}

\author{
Jean-Philippe Julien
}

The Hospital for Sick Children, Toronto, Canada; jean-philippe.julien@sickkids.ca

Half of the world's population is at risk of contracting malaria, which is caused by Plasmodium parasites. Despite extensive public health and biomedical measures, the incidence of malaria continues to rise, with over 200 million cases each year. A highly effective vaccine will likely be required to eradicate malaria; however, current vaccine candidates against Plasmodium falciparum have fallen short in great part because of a lack of understanding of immunity to the parasite at the molecular level. Our recent X-ray crystallography and cryoEM work has revealed structural details of antibody immunity against the malaria parasite. These molecular blueprints of parasite inhibition by antibodies are being leveraged as guides for the design of next-generation subunit vaccines.

Keywords: structural immunology, antibodies, malaria, structure-guided immunogen design 